Louisiana State University

LSU Digital Commons

Faculty Publications

Department of Mathematics

6-1-2008

\title{
Constructions of strict Lyapunov functions for discrete time and hybrid time-varying systems
}

\author{
Michael Malisoff \\ Louisiana State University \\ Frédéric Mazenc \\ INRAE's Occitanie-Montpellier Centre
}

Follow this and additional works at: https://digitalcommons.Isu.edu/mathematics_pubs

\section{Recommended Citation}

Malisoff, M., \& Mazenc, F. (2008). Constructions of strict Lyapunov functions for discrete time and hybrid time-varying systems. Nonlinear Analysis: Hybrid Systems, 2 (2), 394-407. https://doi.org/10.1016/ j.nahs.2006.12.005

This Article is brought to you for free and open access by the Department of Mathematics at LSU Digital Commons. It has been accepted for inclusion in Faculty Publications by an authorized administrator of LSU Digital Commons. For more information, please contact ir@lsu.edu. 


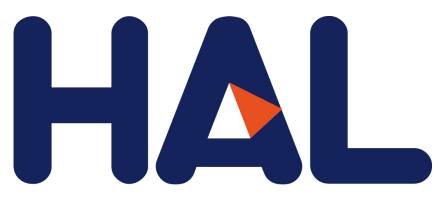

archives-ouvertes

\title{
Constructions of strict Lyapunov functions for discrete time and hybrid time-varying systems
}

\author{
Michael Malisoff, Frédéric Mazenc
}

\section{To cite this version:}

Michael Malisoff, Frédéric Mazenc. Constructions of strict Lyapunov functions for discrete time and hybrid time-varying systems. Nonlinear Analysis: Hybrid Systems, Elsevier, 2008, 2 (2 spécial issue), pp.394-407. 10.1016/j.nahs.2006.12.005 . hal-00857803

\section{HAL Id: hal-00857803 https://hal.inria.fr/hal-00857803}

Submitted on 31 May 2020

HAL is a multi-disciplinary open access archive for the deposit and dissemination of scientific research documents, whether they are published or not. The documents may come from teaching and research institutions in France or abroad, or from public or private research centers.
L'archive ouverte pluridisciplinaire HAL, est destinée au dépôt et à la diffusion de documents scientifiques de niveau recherche, publiés ou non, émanant des établissements d'enseignement et de recherche français ou étrangers, des laboratoires publics ou privés. 


\title{
Constructions of strict Lyapunov functions for discrete time and hybrid time-varying systems
}

\author{
Michael Malisoff ${ }^{\mathrm{a}}$, Frédéric Mazenc ${ }^{\mathrm{b}, *}$ \\ a Department of Mathematics, Louisiana State University, Baton Rouge, LA 70803-4918, United States \\ ${ }^{\mathrm{b}}$ Projet MERE INRIA-INRA, UMR Analyse des Systèmes et Biométrie INRA, 2 pl. Viala, 34060 Montpellier, France
}

Received 6 January 2006; accepted 21 December 2006

\begin{abstract}
We provide explicit closed form expressions for strict Lyapunov functions for time-varying discrete time systems. Our Lyapunov functions are expressed in terms of known nonstrict Lyapunov functions for the dynamics and finite sums of persistency of excitation parameters. This provides a discrete time analog of our previous continuous time Lyapunov function constructions. We also construct explicit strict Lyapunov functions for systems satisfying nonstrict discrete time analogs of the conditions from Matrosov's Theorem. We use our methods to build strict Lyapunov functions for time-varying hybrid systems that contain mixtures of continuous and discrete time evolutions.
\end{abstract}

(C) 2007 Elsevier Ltd. All rights reserved.

Keywords: Strict Lyapunov functions; Discrete and hybrid time-varying systems

\section{Introduction}

The theory of Lyapunov functions plays a fundamental role in modern nonlinear robustness analysis and controller design $[1,3,4,11,14,15,13,16,18]$. In many applications, it is essential to have explicit closed form expressions for a strict Lyapunov function. This is especially the case when one wishes to design stabilizing feedbacks, which are often expressed in terms of the Lie derivatives of Lyapunov functions in the directions of the vector fields that define the system evolution. The classical converse Lyapunov function theorem asserts that systems that are stable in an appropriate sense also admit strict Lyapunov functions [5]. However, the Lyapunov functions provided by the theory are not closed form explicit expressions since they involve infinite sums or improper integrals or optimal control value functions and so do not lend themselves to applications. Moreover, whereas most of the known explicit Lyapunov function constructions are for time-invariant systems, it is well appreciated that time-invariant systems are often inadequate for engineering practice. For example, there are many applications where the dynamics cannot be stabilized by time-invariant feedback but can be stabilized using time-varying controllers [8,19,22,24]. Time-varying systems are also ubiquitous in tracking. While some methods for building Lyapunov functions for time-varying systems are known, general methods for constructing explicit closed form Lyapunov functions for time-varying discrete and hybrid

\footnotetext{
* Corresponding author.

E-mail addresses: malisoff@1su.edu (M. Malisoff), Frederic.Mazenc@ensam.inra.fr (F. Mazenc).
} 
systems are not available. Hybrid systems are ubiquitous in science and engineering [27]. Hence, the construction of explicit Lyapunov functions for time-varying systems presents significant challenges that are of considerable ongoing research interest.

One recently developed and powerful approach to this problem involves constructing strict Lyapunov functions in terms of given nonstrict Lyapunov functions for the system; see for instance $[2,9,16,18]$. By a nonstrict Lyapunov function, we roughly mean a function that is positive definite and radially unbounded and that has a negative semidefinite derivative along all solutions; see Section 2 for precise definitions. The advantage of this strictification approach is that in many applications, a nonstrict Lyapunov function is readily available through backstepping or physical considerations [13]. For continuous time systems, the strictification approach has been applied to rotating rigid bodies, robot manipulators, and other important engineering applications. This suggests the possibility of extending the strictification approach by constructing explicit closed form Lyapunov functions for discrete time time-varying systems or, more generally, hybrid time-varying systems containing both continuous and discrete time evolutions. The purpose of this work is to show that both of the extensions are indeed possible.

In Section 2, we provide the relevant definitions of strict Lyapunov functions and the necessary formalism of hybrid systems, hybrid time domains, and hybrid trajectories. In Section 3, we show how to construct explicit closed form strict Lyapunov functions for time-varying discrete time nonlinear systems in terms of given nonstrict Lyapunov functions. This provides a discrete time analog of [16] as well as a more explicit Lyapunov function construction than the known discrete time constructions that involve infinite sums of persistency of excitation (PE) parameters [20]. We also build Lyapunov functions for time-varying systems under appropriate versions of the assumptions from Matrosov's Theorem thus providing a discrete time analog of the results [18] on continuous time systems satisfying the Matrosov conditions. In Section 4, we merge our results with the known continuous time analogs to construct explicit closed form Lyapunov functions for time-varying hybrid systems, under appropriate hybrid analogs of the PE or Matrosov conditions. To our knowledge, this provides the first general method for explicitly constructing Lyapunov functions for general time-varying nonlinear hybrid systems. In Section 5, we prove our theorems. We provide some examples covered by our results in Section 6, and we close in Section 7 with some remarks about possible extensions.

\section{Definitions, assumptions, and lemmas}

We let $\mathcal{K}_{\infty}$ denote the set of all continuous functions $\rho:[0, \infty) \rightarrow[0, \infty)$ for which (i) $\rho(0)=0$ and (ii) $\rho$ is strictly increasing and unbounded. Note that $\mathcal{K}_{\infty}$ is closed under inverse and composition; i.e., if $\rho_{1}, \rho_{2} \in \mathcal{K}_{\infty}$, then $\rho_{1}^{-1}, \rho_{1} \circ \rho_{2} \in \mathcal{K}_{\infty}$. We let $\mathcal{K} \mathcal{L}$ denote the class of all continuous functions $\beta:[0, \infty) \times[0, \infty) \rightarrow[0, \infty)$ for which (I) $\beta(\cdot, t) \in \mathcal{K}_{\infty}$ for each $t \geq 0$, (II) $\beta(s, \cdot)$ is nonincreasing for each $s \geq 0$, and (III) $\beta(s, t) \rightarrow 0$ as $t \rightarrow+\infty$ for each $s \geq 0$. We let $\mathcal{K} \mathcal{L} \mathcal{L}$ denote the set of all functions $\beta:[0, \infty) \times[0, \infty) \times[0, \infty) \rightarrow[0, \infty)$ such that for each $\bar{t} \geq 0$, the functions $(s, t) \mapsto \beta(s, t, \bar{t})$ and $(s, t) \mapsto \beta(s, \bar{t}, t)$ are of class $\mathcal{K} \mathcal{L}$. When we say that a function $\rho$ is smooth (a.k.a. $C^{1}$ ), we mean it is continuously differentiable, written $\rho \in C^{1}$. (For functions $\rho$ defined on $[0, \infty$ ), we interpret $\rho^{\prime}(0)$ as a one-sided derivative, and continuity of $\rho^{\prime}$ at 0 as one-sided continuity.)

We set $\mathbb{Z}_{\geq 0}=\{0,1,2, \ldots\}$, we let $\mathbb{R}^{n}$ denote the set of all real $n$-tuples, and we use $|\cdot|$ to denote the usual Euclidean norm. We say that a function $\Theta: \mathbb{R}^{n} \times[0, \infty) \times \mathbb{Z}_{\geq 0} \rightarrow \mathbb{R}:(x, t, k) \mapsto \Theta(x, t, k)$ (which may be independent of $t$ or $k$ ) is uniformly state-bounded and write $\Theta \in \mathcal{U S B}$ provided there exists $\mu \in \mathcal{K}_{\infty}$ such that $|\Theta(x, t, k)| \leq \mu(|x|)$ for all $x \in \mathbb{R}^{n}, t \geq 0$, and $k \in \mathbb{Z}_{>0}$. More generally, a vector valued function $H: \mathbb{R}^{n} \times[0, \infty) \times \mathbb{Z}_{\geq 0} \rightarrow \mathbb{R}^{n}:(x, t, k) \mapsto H(x, t, k)$ is of class $\mathcal{U S B}$, written $H \in \mathcal{U S B}$, provided $(x, t, k) \mapsto|H(x, t, k)|$ is of class $\mathcal{U S B}$. Following [13], we also say $\Theta$ is uniformly proper and positive definite $(U P P D)$ and write $\Theta \in \mathcal{U P P} \mathcal{D}$ provided there are $\alpha_{1}, \alpha_{2} \in \mathcal{K}_{\infty}$ such that $\alpha_{1}(|x|) \leq \Theta(x, t, k) \leq \alpha_{2}(|x|)$ for all $x \in \mathbb{R}^{n}, t \in[0, \infty)$, and $k \in \mathbb{Z}_{\geq 0}$. We say $\Theta$ is $\left(\omega_{1}, \omega_{2}\right)$-periodic provided $\omega_{1} \in[0, \infty)$ and $\omega_{2} \in \mathbb{Z}_{\geq 0}$ satisfy

$$
\Theta\left(x, t-\omega_{1}, k-\omega_{2}\right)=\Theta(x, t, k) \quad \forall(x, t, k) \in \mathbb{R}^{n} \times[0, \infty) \times \mathbb{Z}_{\geq 0} .
$$

When $\Theta$ is independent of $t$ (resp., $k$ ), we define $\omega_{2}$-periodicity (resp., $\omega_{1}$-periodicity) analogously. A continuous function defined on a subset of Euclidean space that includes 0 and valued in $(-\infty, 0]$ is negative semi-definite provided it is zero at zero. A continuous function $\alpha$ defined on a subset of Euclidean space and valued in $[0, \infty)$ is positive definite provided $\alpha$ is zero only at zero in which case we also write $\alpha \in \mathcal{P} \mathcal{D}$.

We study the stability properties of the discrete time fully nonlinear time-varying system

$$
x_{k+1}=F\left(x_{k}, k\right)
$$


where we always assume $F \in \mathcal{U S B}$. We also study continuous time time-varying systems

$$
\dot{x}=G(x, t)
$$

where $G \in \mathcal{U S B}$ is locally Lipschitz. We always assume (2) is forward complete, meaning for each $x_{o} \in \mathbb{R}^{n}$ and $t_{o} \geq 0$, there is a unique solution $t \mapsto \phi\left(t, t_{o}, x_{o}\right)$ for (2) defined on $\left[t_{o}, \infty\right)$ that satisfies $\phi\left(t_{o}, t_{o}, x_{o}\right)=x_{o}$. We interpret the solutions of (2) in the generalized Lebesgue almost all (a.a.) sense. We also use $k \mapsto \phi\left(k, k_{o}, x_{o}\right)$ to denote the discrete time solution of (1) satisfying $\phi\left(k_{o}, k_{o}, x_{o}\right)=x_{o}$ whenever this would not lead to confusion. Given a function $V: \mathbb{R}^{n} \times[0, \infty) \times \mathbb{Z}_{\geq 0} \rightarrow \mathbb{R}:(x, t, k) \mapsto V(x, t, k)$, we set

$$
\Delta_{k} V(x, t, k):=V(F(x, k), t, k+1)-V(x, t, k), \quad \mathcal{D} V(x, t, k):=\frac{\partial V}{\partial t}(x, t, k)+\frac{\partial V}{\partial x}(x, t, k) G(x, t)
$$

assuming $(x, t) \mapsto V(x, t, k)$ is also smooth for each $k \in \mathbb{Z}_{\geq 0}$ in the definition of $\mathcal{D} V$. In our analysis of (1), $V$ will generally not depend on $t$ but we need to allow its dependence on $t$ in our discussion of hybrid systems.

Definition 1. (a) Let $V: \mathbb{R}^{n} \times \mathbb{Z}_{\geq 0} \rightarrow \mathbb{R}$ be of class $\mathcal{U P \mathcal { P } D}$. We call $V$ a (strict) Lyapunov function for (1) provided there exists $\alpha_{3} \in \mathcal{P} \mathcal{D}$ such that:

$$
\Delta_{k} V(x, k) \leq-\alpha_{3}(|x|) \quad \forall x \in \mathbb{R}^{n} \& k \in \mathbb{Z}_{\geq 0} .
$$

(b) We say that (1) is globally asymptotically stable $(G A S)$ provided there exists $\beta \in \mathcal{K} \mathcal{L}$ such that for all $x_{o} \in \mathbb{R}^{n}$ and $k_{o} \in \mathbb{Z}_{\geq 0}$, we have $\left|\phi\left(k, k_{o}, x_{o}\right)\right| \leq \beta\left(\left|x_{o}\right|, k-k_{o}\right)$ for all $k \geq k_{o}$.

The corresponding Lyapunov function and GAS definitions for (2) are obtained from Definition 1 by replacing $k$ with $t, \mathbb{Z}_{\geq 0}$ with $[0, \infty)$, and $\Delta_{k} V$ with $\mathcal{D} V$. Notice that we do not require $\alpha_{3}$ to be of class $\mathcal{K}_{\infty}$. For the special case where $\alpha_{3} \in \mathcal{K}_{\infty}$, the existence of a discrete time Lyapunov function $V$ is known to imply that (1) is GAS since then $\Delta_{k} V(x, k) \leq-\alpha(V(x, k))$ everywhere with $\alpha:=\alpha_{3} \circ \alpha_{2}^{-1} \in \mathcal{K}_{\infty}$ and $\alpha_{2}$ as in the $\mathcal{U P P \mathcal { P }}$ condition on $V$ [21, Theorem 8]. Furthermore, by replacing the function $\alpha_{3}(|x|)$ in (3) by the smaller function $\Theta(V(x, k)):=\min \left\{\alpha_{3}(s): \alpha_{2}^{-1}(V(x, k)) \leq s \leq \alpha_{1}^{-1}(V(x, k))\right\}$, we are in a situation where Lemma 15 below applies and straightforwardly implies that one can construct a Lyapunov function satisfying (3) with a new function $\alpha_{3}$ of class $\mathcal{K}_{\infty}$. Combining this fact with the stability result from [21], we get:

Lemma 2. If (1) admits a strict Lyapunov function, then it is GAS.

We also use the following persistency of excitation (PE) notions from $[13,20]$ :

Definition 3. (a) We say that a bounded function $p: \mathbb{Z}_{\geq 0} \rightarrow[0, \infty)$ is of discrete PE type with parameters $l$ and $\delta$ and write $p \in \mathcal{P}_{\text {dis }}(l, \delta)$ provided $l \in \mathbb{Z}_{\geq 0}$ and $\delta>0$ are such that

$$
\sum_{i=k-l}^{k} p(i) \geq \delta \quad \forall k \in \mathbb{Z}_{\geq 0} .
$$

(b) We say that a bounded continuous function $q:[0, \infty) \rightarrow[0, \infty)$ is of continuous PE type with parameters $\tau$ and $\varepsilon$ and write $q \in \mathcal{P}_{\text {cts }}(\tau, \varepsilon)$ provided $\tau \geq 0$ and $\varepsilon>0$ are such that

$$
\int_{t-\tau}^{t} q(r) \mathrm{d} r \geq \varepsilon \quad \forall t \geq 0
$$

(c) We set $\mathcal{P}_{\text {dis }}=\bigcup\left\{\mathcal{P}_{\text {dis }}(l, \delta): l \in \mathbb{Z}_{\geq 0}, \delta>0\right\}$ and $\mathcal{P}_{\text {cts }}=\bigcup\left\{\mathcal{P}_{\text {cts }}(\tau, \varepsilon): \tau \geq 0, \varepsilon>0\right\}$.

Elements of $\mathcal{P}_{\text {dis }}$ and $\mathcal{P}_{\text {cts }}$ are called $P E$ parameters and arise in a variety of contexts, e.g., $q(t)=\sin ^{2}(t)$ as well as cases where $q$ can be null on intervals of arbitrarily large length [13]. The following lemma follows from a simple change of variables, a Fubini Theorem argument (as was used in [13]), and the formula $1+2+\cdots+m=m(m+1) / 2$ :

Lemma 4. Let $l \in \mathbb{Z}_{\geq 0}$, let $\tau, \varepsilon, \delta>0$, and let $p \in \mathcal{P}_{\mathrm{dis}}(l, \delta)$ and $q \in \mathcal{P}_{\mathrm{cts}}(\tau, \varepsilon)$ be bounded above by $\bar{p}$ and $\bar{q}$ respectively. Define the functions $S: \mathbb{Z}_{\geq 0} \rightarrow[0, \infty)$ and $R:[0, \infty) \rightarrow[0, \infty)$ by

$$
S(k):=\sum_{s=k-l}^{k} \sum_{j=s}^{k} p(j), \quad R(t):=\int_{t-\tau}^{t} \int_{z}^{t} q(v) \mathrm{d} v \mathrm{~d} z .
$$


Then $S(k) \leq \bar{p}(l+1)^{2}$ and $R(t) \leq \tau^{2} \bar{q} / 2$ hold for all $k \in \mathbb{Z}_{\geq 0}$ and $t \geq 0$. If $p$ is l-periodic, then so is $S$. If $q$ is $\tau$-periodic, then so is $R$.

We next recall the hybrid system tools developed in [6,7], generalized to time-varying systems. For simplicity, we only consider singleton valued dynamics although our results carry through in the more general setting of difference and differential inclusions. Given sets $C, D \subseteq \mathbb{R}^{n}$ and $F$ and $G$ satisfying the assumptions above, the corresponding hybrid dynamical system is defined to be the formal object

$$
\mathcal{H}:= \begin{cases}\dot{x}=G(x, t), & x \in C \\ x_{k+1}=F\left(x_{k}, k\right), & x_{k} \in D .\end{cases}
$$

A compact hybrid time domain is a subset $E \subset[0, \infty) \times \mathbb{Z}_{\geq 0}$ of the form $\cup_{k=0}^{K-1}\left(\left[t_{k}, t_{k+1}\right] \times\{k\}\right)$ for some finite sequence $0 \leq t_{o} \leq t_{1} \leq \cdots \leq t_{K}$. A hybrid time domain is a set $E \subset[0, \infty) \times \mathbb{Z}_{\geq 0}$ with the property that for all $(T, K) \in E$, the intersection $E \cap([0, T] \times\{0,1, \ldots, K\})$ is a compact hybrid time domain. A hybrid arc is a function $x(t, k)$ defined on a hybrid time domain $\operatorname{dom}(x)$ such that $t \mapsto x(t, k)$ is locally absolutely continuous for each $k$. A hybrid trajectory of (7) is a hybrid arc $x(t, k)$ that satisfies the following:

$\left(\mathrm{S}_{1}\right)$ For all $k \in \mathbb{Z}_{\geq 0}$ and a.a. $t$ such that $(t, k) \in \operatorname{dom}(x)$, we have $x(t, k) \in C$ and $\frac{\partial}{\partial t} x(t, k)=G(x(t, k), t)$.

$\left(\mathrm{S}_{2}\right)$ For all $(t, k) \in \operatorname{dom}(x)$ such that $(t, k+1) \in \operatorname{dom}(x)$, we have $x(t, k) \in D$ and $x(t, k+1)=F(x(t, k), k)$.

Notice that $E$ is a hybrid time domain provided it is a finite or infinite union of sets of the form $\left[t_{k}, t_{k+1}\right] \times\{k\}$ with $\left\{t_{k}\right\}$ nondecreasing in $[0, \infty)$, with a possible additional 'last' set having the form $\left[t_{k}, T\right) \times\{k\}$ with $T$ finite or infinite. To keep our notation simple, we use $\cup_{k \in J}\left(\left[t_{k}, t_{k+1}\right] \times\{k\}\right)$ to denote a generic hybrid time domain with the understanding that (i) either $J=\mathbb{Z}_{\geq 0}$ or $J$ is a finite set of the form $\left\{0,1,2, \ldots, j_{\max }\right\}$ and (ii) $\left[t_{k}, t_{k+1}\right]$ may mean $\left[t_{k}, t_{k+1}\right)$ if $J$ is finite and $k=j_{\max }$. Notice that continuous time solutions of (2) in $C$ and discrete time solutions of (1) in $D$ starting with $k=0$ correspond to hybrid trajectories of (7) that have no switchings between the discrete and continuous evolutions.

Definition 5. (a) Let $V \in \mathcal{U P P \mathcal { D }}$ be $C^{1}$ in $x$ and $t$. We call $V$ a (strict) Lyapunov function for $\mathcal{H}$ provided there exists $\alpha_{3} \in \mathcal{P} \mathcal{D}$ such that the following hold for all $t \geq 0$ and $k \in \mathbb{Z}_{\geq 0}$ :

$$
\Delta_{k} V(x, t, k) \leq-\alpha_{3}(|x|) \quad \forall x \in D ; \quad \mathcal{D} V(x, t, k) \leq-\alpha_{3}(|x|) \quad \forall x \in C .
$$

If, in addition, there is a constant $r>0$ such that

$$
V(F(x, k), t, k+1) \leq \mathrm{e}^{-r} V(x, t, k) \quad \forall x \in D ; \quad \mathcal{D} V(x, t, k) \leq-r V(x, t, k) \quad \forall x \in C,
$$

then we call $V$ an exponential decay Lyapunov function for $\mathcal{H}$. (b) We call $\mathcal{H}$ globally asymptotically stable (GAS) provided there exists $\beta \in \mathcal{K} \mathcal{L} \mathcal{L}$ such that: for each trajectory $x(t, k)$ of $\mathcal{H}$ defined on any hybrid time domain $\cup_{k \in J}\left(\left[t_{k}, t_{k+1}\right] \times\{k\}\right)$, we have $|x(t, k)| \leq \beta\left(\left|x\left(t_{o}, 0\right)\right|, k, t-t_{k}\right)$ for all $k \in J$ and all $t \in\left[t_{k}, t_{k+1}\right]$.

Lemma 6. If $\mathcal{H}$ admits a Lyapunov function, then it is GAS.

To prove this lemma, first note that since $\alpha_{3}$ in (8) is independent of $k$, standard arguments (e.g. those in [23] applied with $\left.a(x):=\alpha_{3}(|x|)\right)$ provide $\beta_{1} \in \mathcal{K} \mathcal{L}$ such that for each hybrid trajectory $x(t, j)$ defined on a hybrid time domain $\cup_{k \in J}\left(\left[t_{k}, t_{k+1}\right] \times\{k\}\right)$ and satisfying any initial condition $x\left(t_{o}, 0\right)=x_{o}$, we have

$$
|x(t, k)| \leq \beta_{1}\left(\left|x\left(t_{k}, k\right)\right|, t-t_{k}\right) \quad \forall k \in J, t \in\left[t_{k}, t_{k+1}\right] .
$$

Similarly, since $\alpha_{3}$ in (8) is independent of $t$, and since we can assume as above that $\alpha_{3} \in \mathcal{K}_{\infty}$, the argument from [21, Theorem 8] provides $\beta_{2} \in \mathcal{K} \mathcal{L}$ such that

$$
\left|x\left(t_{k}, k\right)\right| \leq \beta_{2}\left(\left|x_{o}\right|, k\right) \quad \forall k \in J .
$$

In fact, $\beta_{2}$ can be constructed using the decay conditions from (8) as follows. First note that by arguing as in the proof of Lemma 2 above and replacing $V$ with $\kappa \circ V$ for a suitable function $\kappa \in \mathcal{K}_{\infty}$ in the discrete decay condition without relabeling, we can find $\gamma \in \mathcal{K}_{\infty}$ such that $\Delta_{k} V(x, t, k) \leq-\gamma(V(x, t, k))$ when $x \in D$; see Lemma 15 for the construction of $\kappa$. Since $t \mapsto V(x(t, k), t, k)$ decays on $\left(t_{k}, t_{k+1}\right)$ for each $k$, we get 


$$
\begin{aligned}
V\left(x\left(t_{k+2}, k+1\right), t_{k+2}, k+1\right) \leq V\left(x\left(t_{k+1}, k+1\right), t_{k+1}, k+1\right) & =V\left(F\left(x\left(t_{k+1}, k\right), k\right), t_{k+1}, k+1\right) \text { so } \\
V\left(x\left(t_{k+2}, k+1\right), t_{k+2}, k+1\right)-V\left(x\left(t_{k+1}, k\right), t_{k+1}, k\right) & \leq \Delta_{k} V\left(x\left(t_{k+1}, k\right), t_{k+1}, k\right) \\
& \leq-\gamma\left(V\left(x\left(t_{k+1}, k\right), t_{k+1}, k\right)\right)
\end{aligned}
$$

everywhere. Applying [21, Theorem 8] to the function $k \mapsto V\left(x\left(t_{k+1}, k\right), t_{k+1}, k\right)$ and recalling that $V$ is uniformly proper and positive definite gives $\tilde{\beta}_{2} \in \mathcal{K} \mathcal{L}$ (not depending on the choice of the trajectory) such that $\left|x\left(t_{k+1}, k\right)\right| \leq$ $\tilde{\beta}_{2}\left(\left|x\left(t_{1}, 0\right)\right|, k\right)$ for all $k$. Choosing $\alpha_{1}, \alpha_{2} \in \mathcal{K}_{\infty}$ such that $\alpha_{1}(|x|) \leq V(x, t, k) \leq \alpha_{2}(|x|)$ everywhere, the discrete time decay condition in (8) gives

$$
\begin{aligned}
\left|x\left(t_{k+1}, k\right)\right| & \geq \alpha_{2}^{-1} \circ V\left(x\left(t_{k+1}, k\right), t_{k+1}, k\right) \geq \alpha_{2}^{-1} \circ V\left(x\left(t_{k+1}, k+1\right), t_{k+1}, k+1\right) \\
& \geq \alpha_{2}^{-1} \circ \alpha_{1}\left(\left|x\left(t_{k+1}, k+1\right)\right|\right)
\end{aligned}
$$

for all $k \in J$. Similarly, the continuous time decay condition in (8) gives

$$
\left|x\left(t_{1}, 0\right)\right| \leq \alpha_{1}^{-1} \circ V\left(x\left(t_{1}, 0\right), t_{1}, 0\right) \leq \alpha_{1}^{-1} \circ V\left(x\left(t_{o}, 0\right), t_{o}, 0\right) \leq \alpha_{1}^{-1} \circ \alpha_{2}\left(\left|x_{o}\right|\right) .
$$

We can therefore satisfy (11) by taking $\beta_{2}(s, k):=\alpha_{1}^{-1} \circ \alpha_{2} \circ \tilde{\beta}_{2}\left(\alpha_{1}^{-1} \circ \alpha_{2}(s), k\right)+s /(k+1)$, where the additional term $s /(k+1)$ is used to account for the case $k=0$. Combining (10) and (11) shows we can satisfy the requirements of Lemma 6 using $\beta(s, t, k)=\beta_{1}\left(\beta_{2}(s, k), t\right)$.

\section{Statement of results on discrete time systems}

\subsection{Strictifying persistence of excitation (PE) decay estimates}

We begin by constructing explicit closed form Lyapunov functions for discrete time systems in terms of nonstrict Lyapunov functions and appropriate PE parameters $p \in \mathcal{P}_{\text {dis. }}$. For an alternative construction, involving infinite sums of $\mathrm{PE}$ parameter values, see [20]. We prove the following in Section 5:

Theorem 7. Let $l \in \mathbb{Z}_{\geq 0}, \delta>0, p \in \mathcal{P}_{\text {dis }}(l, \delta), V \in \mathcal{U P} \mathcal{P} \mathcal{D}$, and $\Theta \in \mathcal{P} \mathcal{D}$ satisfy

$$
\Delta_{k} V(x, k) \leq-p(k+1) \Theta(V(x, k)) \quad \forall x \in \mathbb{R}^{n} \& k \in \mathbb{Z}_{\geq 0} .
$$

Then one can construct $\kappa, \gamma \in \mathcal{K}_{\infty}$ such that

$$
U(x, k):=\kappa(V(x, k))+\frac{\gamma(V(x, k))}{4(l+1)} \sum_{s=k-l}^{k} \sum_{j=s}^{k} p(j)
$$

is a strict Lyapunov function for (1), so (1) is GAS. If $p$ and $V$ are also both l-periodic in $k$, then so is $U$.

Remark 8. A key feature in (12) is that the PE condition on $p$ allows $p(k+1)=0$ for some values of $k$ in which case we could have $\Delta_{k} V(x, k)=0$. An additional novel feature of Theorem 7 is that we do not require the gain function $\Theta$ in (12) to be of class $\mathcal{K}_{\infty}$. This properness of the gain function was required in [13,20]. Our proof of Theorem 7 will show that we can take $\kappa(s) \equiv s$ if $\Theta \in \mathcal{K}_{\infty}$.

\subsection{Lyapunov function constructions under Matrosov conditions}

Recall the definitions of $\mathcal{U P P D}$ and $\mathcal{U S B}$ from Section 2 . We explicitly construct a Lyapunov function for discrete time systems (1) satisfying the following analog of the Matrosov Theorem conditions from [18]:

Assumption 9. There exist $V_{1}: \mathbb{R}^{n} \times \mathbb{Z}_{\geq 0} \rightarrow[0, \infty)$ of class $\mathcal{U P P} \mathcal{D}, V_{2}: \mathbb{R}^{n} \times \mathbb{Z}_{\geq 0} \rightarrow \mathbb{R}$ of class $\mathcal{U S B}$, a function $\phi_{2} \in \mathcal{K}_{\infty}$, nonnegative functions $N_{1}, N_{2} \in \mathcal{U S B}$, a function $\chi: \mathbb{R}^{n} \times[0, \infty) \times \mathbb{Z}_{\geq 0} \rightarrow \mathbb{R}$, a positive increasing function $\phi_{1}$, a positive definite function $W$, and $p \in \mathcal{P}_{\text {dis }}$ such that

$$
\begin{aligned}
& \Delta_{k} V_{1}(x, k) \leq-N_{1}(x, k), \\
& \Delta_{k} V_{2}(x, k) \leq-N_{2}(x, k)+\chi\left(x, N_{1}(x, k), k\right), \\
& \left|\chi\left(x, N_{1}(x, k), k\right)\right| \leq \phi_{1}(|x|) \phi_{2}\left(N_{1}(x, k)\right), \quad \text { and } \quad N_{1}(x, k)+N_{2}(x, k) \geq p(k+1) W(x)
\end{aligned}
$$

hold for all $x \in \mathbb{R}^{n}$ and $k \in \mathbb{Z}_{\geq 0}$. 
Notice that we allow $V_{2}$ to take both positive and negative values. In Section 5, we prove:

Theorem 10. If (1) satisfies Assumption 9, then one can construct an explicit strict Lyapunov function for (1). In particular, (1) is GAS.

\section{Statement of results on hybrid systems}

\subsection{Hybrid persistency of excitation estimates}

We next extend Theorem 7 to hybrid systems. To keep the exposition simple, we assume the gain functions $\Theta$ in (12) and its continuous analog are $\Theta(s)=s$, but the extension to general positive definite $\Theta$ can be done using similar arguments. We prove the following in Section 5:

Theorem 11. Let $V \in \mathcal{U P P \mathcal { D }}$ be $C^{1}$ in $x$ and $t$. Consider the hybrid system $\mathcal{H}$ in (7), and let $\delta, \varepsilon, \tau>0$ and $l \in \mathbb{Z}_{\geq 0}$ be given. Assume there exist $r \in \mathcal{P}_{\mathrm{dis}}(l, \delta)$ and $q \in \mathcal{P}_{\mathrm{cts}}(\tau, \varepsilon)$ such that

$$
V(F(x, k), t, k+1) \leq \mathrm{e}^{-r(k+1)} V(x, t, k) \quad \forall x \in D ; \quad \mathcal{D} V(x, t, k) \leq-q(t) V(x, t, k) \quad \forall x \in C
$$

hold for all $t \geq 0$ and $k \in \mathbb{Z}_{\geq 0}$. Then

$$
V^{\sharp}(x, t, k)=\left[2+\frac{1}{4(l+1)} \sum_{s=k-l}^{k} \sum_{j=s}^{k}\left(1-\mathrm{e}^{-r(j)}\right)+\frac{1}{\tau} \int_{t-\tau}^{t} \int_{z}^{t} q(v) \mathrm{d} v \mathrm{~d} z\right] V(x, t, k)
$$

is an exponential decay Lyapunov function for $\mathcal{H}$ which is therefore GAS. If in addition $V$ is $(\tau, l)$-periodic and $r$ and $q$ are l-periodic and $\tau$-periodic respectively, then $V^{\sharp}$ is also $(\tau, l)$-periodic.

Remark 12. The preceding theorem covers continuous dynamics (by taking $D=\emptyset$ and $C=\mathbb{R}^{n}$ with the understanding that the term involving the double sum in $V^{\sharp}$ is not present) and discrete dynamics (by taking $C=\emptyset$ and $D=\mathbb{R}^{n}$ in which case the term involving the double integral in $V^{\sharp}$ is not present). See [6] for an alternative, nonexplicit construction of a Lyapunov function for time-invariant hybrid systems.

\subsection{Hybrid systems satisfying Matrosov conditions}

We next extend Theorem 10 to hybrid systems that satisfy the following analog of Assumption 9.

Assumption 13. There exist $V_{1} \in \mathcal{U P P \mathcal { D }}$ and $V_{2} \in \mathcal{U S B}$ that are $C^{1}$ in $(x, t)$, nonnegative $N_{1}, N_{2} \in \mathcal{U} \mathcal{S B}$, a function $\chi: \mathbb{R}^{n} \times[0, \infty)^{2} \times \mathbb{Z}_{\geq 0} \rightarrow \mathbb{R}$, a positive increasing $\phi_{1}$, and a positive definite function $W, p \in \mathcal{P}_{\text {dis }}$, $\phi_{2} \in \mathcal{K}_{\infty}$, and $q \in \mathcal{P}_{\text {cts }}$ such that

1. For all $x \in D$, we have $\Delta_{k} V_{1}(x, t, k) \leq-N_{1}(x, t, k), \Delta_{k} V_{2}(x, t, k) \leq-N_{2}(x, t, k)+\chi\left(x, N_{1}(x, t, k), t, k\right)$, and $N_{1}(x, t, k)+N_{2}(x, t, k) \geq p(k+1) W(x)$.

2. For all $x \in C$, we have $\mathcal{D} V_{1}(x, t, k) \leq-N_{1}(x, t, k), \mathcal{D} V_{2}(x, t, k) \leq-N_{2}(x, t, k)+\chi\left(x, N_{1}(x, t, k), t, k\right)$, and $N_{1}(x, t, k)+N_{2}(x, t, k) \geq q(t) W(x)$.

3. For all $x \in \mathbb{R}^{n}$, we have $\left|\chi\left(x, N_{1}(x, t, k), t, k\right)\right| \leq \phi_{1}(|x|) \phi_{2}\left(N_{1}(x, t, k)\right)$

hold for all $t \geq 0$ and $k \in \mathbb{Z}_{\geq 0}$.

Assumption 13 simply means the discrete and continuous parts of $\mathcal{H}$ satisfy the appropriate discrete and continuous Matrosov conditions. It reduces to Assumption 9 for discrete systems when $C=\emptyset$ and $D=\mathbb{R}^{n}$ in which case its condition 2 holds vacuously. Notice that we again do not require $V_{2}$ to be nonnegative. In Section 5, we prove:

Theorem 14. If $\mathcal{H}$ satisfies Assumption 13, then one can construct an explicit closed form strict Lyapunov function for $\mathcal{H}$. In particular, $\mathcal{H}$ is GAS. 


\section{Proofs of theorems}

\subsection{Results on discrete systems}

\subsubsection{Proof of Theorem 7}

By minorizing $\Theta \in \mathcal{P D}$ without relabeling as in [17], we assume in the following that $\Theta \in C^{1}$ is nondecreasing on $[0,1]$ and nonincreasing on $[1, \infty)$. The next technical lemma allows us to assume that $\Theta \in \mathcal{K}_{\infty}$ in (12):

Lemma 15. Let $\Theta \in \mathcal{P} \mathcal{D}$ be as above and $p \in \mathcal{P}_{\text {dis. }}$ Define $\mu:[0, \infty) \rightarrow[1, \infty), \kappa$, and $\chi$ by

$$
\kappa(r):=2 \int_{0}^{r} \mu(z) \mathrm{d} z, \quad \chi(r):=\Theta(2 r) \mu(r), \quad \text { and } \quad \mu(r)= \begin{cases}1+4 r^{2}, & 0 \leq r \leq 1 / 2 \\ \frac{4 \Theta(1) r}{\Theta(2 r)}, & 1 / 2 \leq r<\infty .\end{cases}
$$

Let $v \in \mathcal{U P P \mathcal { D }}$ satisfy $\Delta_{k} v(x, k) \leq-p(k+1) \Theta(v(x, k))$ for all $x \in \mathbb{R}^{n}$ and $k \in \mathbb{Z}_{\geq 0}$. Then $\kappa \in \mathcal{K}_{\infty} \cap C^{1}, \chi \in \mathcal{K}_{\infty}$, and $V:=\kappa(v) \in \mathcal{U P P} \mathcal{D}$ satisfies

$$
\Delta_{k} V(x, k) \leq-p(k+1) \gamma(V(x, k)) \quad \forall x \in \mathbb{R}^{n} \& k \in \mathbb{Z}_{\geq 0},
$$

where $\gamma \in \mathcal{K}_{\infty}$ is defined by $\gamma(s):=\chi\left(\kappa^{-1}(s) / 2\right)$.

To prove Lemma 15, fix $x \in \mathbb{R}^{n}$ and $k \in \mathbb{Z}_{\geq 0}$ and apply the Fundamental Theorem of Calculus to $s \mapsto \mathcal{F}(s):=$ $\kappa(s v(F(x, k), k+1)+(1-s) v(x, k))$ to write $\Delta_{k} V(x, k)=\mathcal{F}(1)-\mathcal{F}(0)=\int_{0}^{1} \mathcal{F}^{\prime}(s) \mathrm{d} s$ and so also

$$
\begin{aligned}
\Delta_{k} V(x, k) & =\left[\int_{0}^{1} \kappa^{\prime}(s v(F(x, k), k+1)+(1-s) v(x, k)) \mathrm{d} s\right][v(F(x, k), k+1)-v(x, k)] \\
& \leq-p(k+1)\left[\int_{0}^{1} \kappa^{\prime}(s v(F(x, k), k+1)+(1-s) v(x, k)) \mathrm{d} s\right] \Theta(v(x, k)) \\
& \leq-p(k+1)\left[\int_{0}^{1} \kappa^{\prime}((1-s) v(x, k)) \mathrm{d} s\right] \Theta(v(x, k)) \\
& \leq-p(k+1)\left[\int_{0}^{1 / 2} \kappa^{\prime}\left(\frac{1}{2} v(x, k)\right) \mathrm{d} s\right] \Theta(v(x, k))=-p(k+1) \mu\left(\frac{1}{2} v(x, k)\right) \Theta(v(x, k))
\end{aligned}
$$

where the first inequality holds because $\kappa$ is nondecreasing and the other inequalities used the fact that $\kappa^{\prime}$ is nondecreasing. The lemma now follows from our choices of $\gamma$ and $\chi$.

We can therefore assume that $V$ satisfies (17) with $\gamma \in \mathcal{K}_{\infty}$, possibly by replacing $V$ with $\kappa(V)$ for $\kappa \in \mathcal{K}_{\infty}$ defined in (16). Defining $S(k)$ as in (6) and defining $U$ by (13) with $\kappa(s) \equiv s$ therefore gives

$$
\begin{aligned}
\Delta_{k} U(x, k) & =V(F(x, k), k+1)+\frac{S(k+1)}{4(l+1)} \gamma(V(F(x, k), k+1))-V(x, k)-\frac{S(k)}{4(l+1)} \gamma(V(x, k)) \\
& =\Delta_{k} V(x, k)+\frac{1}{4(l+1)} S(k+1) \Delta_{k}(\gamma \circ V)(x, k)+\frac{1}{4(l+1)} \gamma(V(x, k))[S(k+1)-S(k)] \\
& \leq \Delta_{k} V(x, k)+\frac{1}{4(l+1)} \gamma(V(x, k))[S(k+1)-S(k)],
\end{aligned}
$$

where the last inequality holds because $\gamma$ is increasing, so $\Delta_{k}(\gamma \circ V)(x, k) \leq 0$. Note that

$$
\begin{aligned}
S(k+1)-S(k) & =\sum_{s=k+1}^{k} \sum_{j=s}^{k} p(j)+(l+1) p(k+1)-\sum_{s=k-l}^{k} \sum_{j=s}^{k} p(j) \\
& =\sum_{s=k-l}^{k} \sum_{j=s}^{k} p(j)-\sum_{j=k-l}^{k} p(j)+(l+1) p(k+1)-\sum_{s=k-l}^{k} \sum_{j=s}^{k} p(j)
\end{aligned}
$$




$$
=-\sum_{j=k-l}^{k} p(j)+(l+1) p(k+1) .
$$

Substituting (19) into (18) gives

$$
\begin{aligned}
\Delta_{k} U(x, k) & \leq \Delta_{k} V(x, k)+\frac{1}{4(l+1)} \gamma(V(x, k))\left((l+1) p(k+1)-\sum_{j=k-l}^{k} p(j)\right) \\
& \leq \Delta_{k} V(x, k)+\frac{p(k+1) \gamma(V(x, k))}{4}-\frac{\gamma(V(x, k))}{4(l+1)} \sum_{j=k-l}^{k} p(j) \leq-\frac{\delta}{4(l+1)} \gamma(V(x, k)),
\end{aligned}
$$

where the last inequality follows from the PE property of $p$ and (17). This, the fact that $V \in \mathcal{U P P \mathcal { D }}$, and the global boundedness of $S(k)$ from Lemma 4 shows that $U$ is a Lyapunov function for (1). Therefore, (1) is GAS, by Lemma 2. The assertion in the theorem about periodicity follows from Lemma 4 and the formula for $U$, so this proves our theorem.

\subsubsection{Proof of Theorem 10}

Let $V_{3}=V_{1}+V_{2}$, and let $\alpha_{1}, \alpha_{2} \in \mathcal{K}_{\infty}$ satisfy the UPPD requirements for $V_{1}$. In the rest of the proof, all inequalities should be interpreted as holding globally unless otherwise indicated. We also leave out the argument $(x, k)$ of some of our $\mathcal{U S B}$ functions when this would not lead to confusion. It follows from Assumption 9 that we can determine a positive definite function $\lambda$ such that

$$
\Delta_{k} V_{3}(x, k) \leq-p(k+1) W(x)+\phi_{1}(|x|) \phi_{2}\left(N_{1}(x, k)\right) \leq-p(k+1) \lambda\left(V_{1}(x, k)\right)+\phi_{1}(|x|) \phi_{2}\left(N_{1}(x, k)\right)
$$

e.g. $\lambda(s)=\min \left\{W(x): x \in \mathbb{R}^{n}, \alpha_{1}(|x|) \leq s \leq \alpha_{2}(|x|)\right\}$ (which is positive definite because $W \in \mathcal{P} \mathcal{D}$ ). By minorizing $\lambda$ as necessary as in [17], we can assume it is $C^{1}$, nondecreasing on [0,1/2] and nonincreasing on [1/2, $\infty$ ). The proof of Lemma 15 above with $\Theta(r):=\lambda(r / 2)$ provides an increasing continuous function $k_{1}:[0, \infty) \rightarrow[1, \infty)$ such that $\Lambda_{1}(s):=k_{1}(s) \lambda(s)$ is of class $\mathcal{K}_{\infty}$. Let $V_{4}=k_{1}\left(V_{1}\right) V_{3}$. Then (21) gives

$$
\begin{aligned}
\Delta_{k} V_{4}(x, k)= & {\left[k_{1}\left(V_{1}(F(x, k), k+1)\right)-k_{1}\left(V_{1}(x, k)\right)\right] V_{3}(F(x, k), k+1) } \\
& +k_{1}\left(V_{1}(x, k)\right)\left[V_{3}(F(x, k), k+1)-V_{3}(x, k)\right] \\
\leq & {\left[k_{1}\left(V_{1}(F(x, k), k+1)\right)-k_{1}\left(V_{1}(x, k)\right)\right] V_{3}(F(x, k), k+1) } \\
& -k_{1}\left(V_{1}(x, k)\right) p(k+1) \lambda\left(V_{1}(x, k)\right)+k_{1}\left(V_{1}(x, k)\right) \phi_{1}(|x|) \phi_{2}\left(N_{1}(x, k)\right) .
\end{aligned}
$$

Since $F, V_{3} \in \mathcal{U S B}$ and $V_{1} \in \mathcal{U P P \mathcal { D }}$, we get continuous increasing positive functions $\Gamma$ and $\Lambda_{2}$ such that

$$
\Delta_{k} V_{4}(x, k) \leq\left[-\Delta_{k} V_{1}(x, k)\right] \Gamma\left(V_{1}(x, k)\right)-p(k+1) \Lambda_{1}\left(V_{1}(x, k)\right)+\Lambda_{2}\left(V_{1}(x, k)\right) \phi_{2}\left(N_{1}(x, k)\right)
$$

e.g. by first finding an increasing positive function $\tilde{\alpha}$ such that $\left|k_{1}^{\prime}(r)\right| \leq \tilde{\alpha}(r)$. Define $k_{2} \in \mathcal{K}_{\infty}$ by $k_{2}(s)=s \Gamma(s)$. Since $k_{2}(s) / s$ is increasing, we have $k_{2}(b)-k_{2}(a) \geq(b-a) \Gamma(b)$ when $b \geq a \geq 0$ (since $\left(k_{2}(b)-k_{2}(a)\right.$ ) $(b-a)^{-1} \geq k_{2}(b) b^{-1}$ when $\left.b>a \geq 0\right)$. Hence by choosing $a=V_{1}(F(x, k), k+1)$ and $b=V_{1}(x, k)$, we get $\Delta_{k}\left(k_{2} \circ V_{1}\right)(x, k) \leq \Delta_{k} V_{1}(x, k) \Gamma\left(V_{1}(x, k)\right)$ everywhere. Therefore, by adding a $\mathcal{K}_{\infty}$ function to $k_{2}$ as necessary, we can assume $V_{5}:=V_{4}+k_{2}\left(V_{1}\right) \in \mathcal{U P \mathcal { P }}$ and satisfies

$$
\Delta_{k} V_{5}(x, k) \leq-p(k+1) \Lambda_{1}\left(V_{1}(x, k)\right)+\Lambda_{2}\left(V_{1}(x, k)\right) \phi_{2}\left(N_{1}(x, k)\right) .
$$

Arguing as in the proof of Theorem 7 except with $\gamma$ replaced by $\Lambda_{1}$ provides $V_{6} \in \mathcal{U P \mathcal { P } D}$ such that

$$
\Delta_{k} V_{6}(x, k) \leq-\frac{\delta}{4(l+1)} \Lambda_{1}\left(V_{1}(x, k)\right)+\Lambda_{2}\left(V_{1}(x, k)\right) \phi_{2}\left(N_{1}(x, k)\right) .
$$

By arguing as in [17, Section IV.A], we can select $k_{3} \in C^{1} \cap \mathcal{P D}$ so that $\left|k_{3}^{\prime}(s)\right| \leq 1$ for all $s \geq 0$ and

$$
k_{3}(r) \leq \phi_{2}^{-1}\left(\frac{\delta}{8(l+1)} \frac{\Lambda_{1}(r)}{1+\Lambda_{2}(r)}\right) \frac{1}{1+\Lambda_{2}(r)}, \quad \text { hence } \phi_{2}\left(k_{3}\left(V_{1}\right) \Lambda_{2}\left(V_{1}\right)\right) \Lambda_{2}\left(V_{1}\right) \leq \frac{\delta}{8(l+1)} \Lambda_{1}\left(V_{1}\right)
$$


everywhere. Choose $\mu_{F}, \alpha_{6} \in \mathcal{K}_{\infty}$ such that $V_{6}(x, k) \leq \alpha_{6}(|x|)$ and $|F(x, k)| \leq \mu_{F}(|x|)$ everywhere. Arguing as above (with $\Gamma$ replaced by $\alpha_{6} \circ \mu_{F} \circ \alpha_{1}^{-1}$ ) and recalling that $\Delta_{k} V_{1}(x, k) \leq 0$ provides $k_{4} \in \mathcal{K}_{\infty}$ such that

$$
\Delta_{k}\left(k_{4} \circ V_{1}\right)(x, k) \leq \alpha_{6} \circ \mu_{F} \circ \alpha_{1}^{-1} \circ V_{1}(x, k) \Delta_{k} V_{1}(x, k) \leq \alpha_{6}\left(\mu_{F}(|x|)\right) \Delta_{k} V_{1}(x, k)
$$

everywhere. Since $\left|k_{3}^{\prime}(s)\right| \leq 1$ for all $s \geq 0$, we have

$$
\left[\Delta_{k}\left(k_{3} \circ V_{1}\right)(x, k)\right] V_{6}(F(x, k), k+1) \leq\left[-\Delta_{k} V_{1}(x, k)\right] V_{6}(F(x, k), k+1) \leq-\alpha_{6}\left(\mu_{F}(|x|)\right) \Delta_{k} V_{1}(x, k)
$$

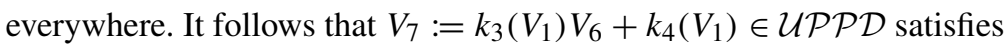

$$
\begin{aligned}
\Delta_{k} V_{7}(x, k) & =\left[\Delta_{k}\left(k_{3} \circ V_{1}\right)(x, k)\right] V_{6}(F(x, k), k+1)+k_{3}\left(V_{1}(x, k)\right) \Delta_{k} V_{6}(x, k)+\Delta_{k}\left(k_{4} \circ V_{1}\right)(x, k) \\
& \leq-\frac{\delta}{4(l+1)} k_{3}\left(V_{1}\right) \Lambda_{1}\left(V_{1}\right)+k_{3}\left(V_{1}\right) \Lambda_{2}\left(V_{1}\right) \phi_{2}\left(N_{1}(x, k)\right) .
\end{aligned}
$$

Next note that for all functions $\mu \in \mathcal{K}_{\infty}$,

$$
\Delta_{k} V_{7} \leq-\frac{\delta}{4(l+1)} k_{3}\left(V_{1}\right) \Lambda_{1}\left(V_{1}\right)+\mu\left(k_{3}\left(V_{1}\right) \Lambda_{2}\left(V_{1}\right)\right) k_{3}\left(V_{1}\right) \Lambda_{2}\left(V_{1}\right)+\mu^{-1}\left(\phi_{2}\left(N_{1}(x, k)\right)\right) \phi_{2}\left(N_{1}(x, k)\right) .
$$

(The fact that $a b \leq \mu(a) a+\mu^{-1}(b) b$ for all $a, b \geq 0$ follows by separately considering the cases where $\mu(a) \geq b$ and $\mu^{-1}(b) \geq a$.) Choosing $\mu=\phi_{2}$ in (28) gives

$$
\begin{aligned}
\Delta_{k} V_{7} & \leq-\frac{\delta}{4(l+1)} k_{3}\left(V_{1}\right) \Lambda_{1}\left(V_{1}\right)+\phi_{2}\left(k_{3}\left(V_{1}\right) \Lambda_{2}\left(V_{1}\right)\right) k_{3}\left(V_{1}\right) \Lambda_{2}\left(V_{1}\right)+N_{1}(x, k) \phi_{2}\left(N_{1}\right) \\
& \leq-\frac{\delta}{8(l+1)} k_{3}\left(V_{1}\right) \Lambda_{1}\left(V_{1}\right)+N_{1}(x, k) \phi_{2}\left(N_{1}\right) \quad(\text { by }(26)) .
\end{aligned}
$$

Therefore, since $N_{1} \in \mathcal{U S B}$ and $\phi_{2} \in \mathcal{K}_{\infty}$, a suitable function $\phi_{3} \in \mathcal{K}_{\infty}$ gives

$$
\Delta_{k} V_{7} \leq-\frac{\delta}{8(l+1)} k_{3}\left(V_{1}\right) \Lambda_{1}\left(V_{1}\right)+N_{1}(x, k) \phi_{3}\left(V_{1}\right) .
$$

Arguing as in the construction of $k_{2}$ above (but with $\Gamma$ replaced by $\left.\phi_{3}\right)$ provides $k_{5} \in \mathcal{K}_{\infty}$ such that $\Delta_{k}\left(k_{5} \circ V_{1}\right)(x, k) \leq$ $\Delta_{k} V_{1}(x, k) \phi_{3}\left(V_{1}(x, k)\right) \leq-N_{1}(x, k) \phi_{3}\left(V_{1}(x, k)\right)$. Hence, $V_{8}:=V_{7}+k_{5}\left(V_{1}\right) \in \mathcal{U P} \mathcal{P} \mathcal{D}$ satisfies

$$
\Delta_{k} V_{8}(x, k) \leq-\frac{\delta}{8(l+1)} k_{3}\left(V_{1}(x, k)\right) \Lambda_{1}\left(V_{1}(x, k)\right) \leq-\alpha_{3}(|x|),
$$

where

$$
\alpha_{3}(s):=\frac{\delta}{8(l+1)} \min \left\{k_{3}(u) \Lambda_{1}(u): \alpha_{1}(s) \leq u \leq \alpha_{2}(s)\right\} .
$$

Since $\alpha_{3} \in \mathcal{P} \mathcal{D}, V_{8}$ satisfies the requirements of the theorem. This and Lemma 2 proves the theorem.

\subsection{Results for hybrid systems}

\subsubsection{Proof of Theorem 11}

For each $k \in \mathbb{Z}_{\geq 0}$, let $V_{\text {cts }}(x, t, k)$ denote the continuous time strictification of $V$ obtained in [13] for the nonstrictness parameter $q \in \mathcal{P}_{\text {cts }}$. Thus,

$$
V_{\mathrm{cts}}(x, t, k):=\left[1+\frac{1}{\tau} \int_{t-\tau}^{t} \int_{z}^{t} q(v) \mathrm{d} v \mathrm{~d} z\right] V(x, t, k) .
$$

The results from [13] show that $\mathcal{D} V_{\text {cts }}(x, t, k) \leq-(\varepsilon / \tau) V_{\text {cts }}(x, t, k)$ for all $x \in C, t \geq 0$, and $k \in \mathbb{Z}_{\geq 0}$. We next rewrite the first decay condition in (14) as

$$
V(F(x, k), t, k+1)-V(x, t, k) \leq-p(k+1) V(x, t, k), \quad \forall x \in D, t \in[0, \infty), k \in \mathbb{Z}_{\geq 0}
$$


where $k \mapsto p(k):=1-\mathrm{e}^{-r(k)}$ is again of PE type. For each $t \geq 0$, let $V_{\text {dis }}(x, t, k)$ be the strictification

$$
V_{\mathrm{dis}}(x, t, k):=\left[1+\frac{1}{4(l+1)} \sum_{s=k-l}^{k} \sum_{j=s}^{k} p(j)\right] V(x, t, k)
$$

of $V$ from Theorem 7. The proof of Theorem 7 shows we can take $\kappa(s) \equiv \gamma(s) \equiv s$, and therefore also

$$
\Delta_{k} V_{\mathrm{dis}}(x, t, k)=V_{\mathrm{dis}}(F(x, k), t, k+1)-V_{\mathrm{dis}}(x, t, k) \leq-\frac{\delta}{4(l+1)} V_{\mathrm{dis}}(x, t, k) \quad \forall t \in[0, \infty), k \in \mathbb{Z}_{\geq 0}
$$

for all $x \in D$. By enlarging $l$ from the PE assumption as necessary, we can assume $\delta<l$. It follows that the discrete decay condition in (14) holds with $V$ replaced by $V_{\text {dis }}$ and with the constant

$$
r(k) \equiv \ln \left(\frac{4(l+1)}{4(l+1)-\delta}\right)>0 .
$$

Since $\mathcal{D} V \leq 0$ on $C$ and $\Delta_{k} V \leq 0$ on $D$, we have $\mathcal{D} V_{\text {dis }} \leq 0$ on $C$ and $\Delta_{k} V_{\text {cts }} \leq 0$ on $D$. The uniform boundedness of $S(k)$ and $\int_{t-\tau}^{t} \int_{z}^{t} q(v) \mathrm{d} v \mathrm{~d} z$ from Lemma 4 provides constants $r_{c}, r_{d}>0$ such that

$$
V_{\text {cts }}(x, t, k) \leq r_{c} V_{\text {dis }}(x, t, k) \leq r_{d} V_{\text {cts }}(x, t, k)
$$

everywhere. One therefore easily checks that $V^{\sharp}(x, t, k):=V_{\text {cts }}(x, t, k)+V_{\text {dis }}(x, t, k)$ as given by (15) is an exponential decay Lyapunov function for the hybrid dynamic $\mathcal{H}$. The periodicity assertion follows as before from Lemma 4, so the result follows from Lemma 6.

\subsubsection{Proof of Theorem 14}

For each $t \geq 0$ we apply the first part of the proof of Theorem 10 to the functions $(x, k) \mapsto V_{1}(x, t, k)$ and $(x, k) \mapsto V_{2}(x, t, k)$ to get $V_{5}$ that satisfies

$$
\Delta_{k} V_{5}(x, t, k) \leq-p(k+1) \Lambda_{1}\left(V_{1}(x, t, k)\right)+\Lambda_{2}\left(V_{1}(x, t, k)\right) \phi_{2}\left(N_{1}(x, t, k)\right) \quad \forall x \in D, t \geq 0, k \in \mathbb{Z}_{\geq 0} .
$$

This can be done with $\Lambda_{1} \in C^{1}$ and $\Lambda_{2}$ independent of $t$. For each $k \in \mathbb{Z}_{\geq 0}$, we next apply the continuous time analog of the preceding argument (which is almost exactly the same except with $\Delta_{k} V_{i}$ replaced by $\mathcal{D} V_{i}$ for $i=1,2, \ldots, 5$, as discussed in the Appendix below) to get a continuous version $V_{5}^{\text {cts }}$ of $V_{5}$ that satisfies

$$
\mathcal{D} V_{5}^{\text {cts }}(x, t, k) \leq-q(t) \Lambda_{1}\left(V_{1}(x, t, k)\right)+\Lambda_{2}\left(V_{1}(x, t, k)\right) \phi_{2}\left(N_{1}(x, t, k)\right) \quad \forall x \in C, t \geq 0, k \in \mathbb{Z}_{\geq 0} .
$$

In fact, by enlarging $k_{2}$ as necessary (e.g., by enlarging $\Gamma$ in the discrete version of the proof), we can assume $V_{5}^{\text {cts }}$ and $V_{5}$ have the same formula. Applying the strictification method from Theorem 7 to $V_{5}$ produces

$$
V_{6}^{\mathrm{dis}}(x, t, k):=V_{5}(x, t, k)+\frac{1}{4(l+1)} S(k) \Lambda_{1}\left(V_{5}(x, t, k)\right)
$$

that satisfies (25) with $V_{6}$ replaced by $V_{6}^{\text {dis }}$ and with $V_{1}$ and $N_{1}$ now also depending on $t$. Similarly, we apply the continuous time strictification from [13] (as in the proof of Theorem 11) to $V_{5}^{\text {cts }}$ to get

$$
V_{6}^{\mathrm{cts}}(x, t, k):=V_{5}^{\mathrm{cts}}(x, t, k)+\frac{1}{\tau}\left[\int_{t-\tau}^{t} \int_{z}^{t} q(v) \mathrm{d} v \mathrm{~d} z\right] \Lambda_{1}\left(V_{5}^{\mathrm{cts}}(x, t, k)\right)
$$

that satisfies $\mathcal{D} V_{6}^{\mathrm{cts}}(x, t, k) \leq-\Lambda_{1}\left(V_{1}(x, t, k)\right)+\Lambda_{2}\left(V_{1}(x, t, k)\right) \phi_{2}\left(N_{1}(x, t, k)\right)$ when $x \in C$, possibly by reducing $\Lambda_{1}$ and increasing $\Lambda_{2} \in \mathcal{K}_{\infty}$ without relabeling. Setting $V_{6}=V_{6}^{\text {cts }}+V_{6}^{\text {dis }}$, and assuming without loss of generality that $1>\delta /\{4(l+1)\}$ (by enlarging $l$ without relabeling as before), it follows from the fact that $V_{5}^{\text {cts }}$ and $V_{5}$ have the same formula that we can enlarge $\Lambda_{2}$ sufficiently so that

$$
\begin{array}{ll}
\Delta_{k} V_{6}(x, t, k) \leq-\frac{\delta}{4(l+1)} \Lambda_{1}\left(V_{1}(x, t, k)\right)+\Lambda_{2}\left(V_{1}(x, t, k)\right) \phi_{2}\left(N_{1}(x, t, k)\right) & \forall x \in D \\
\mathcal{D} V_{6}(x, t, k) \leq-\frac{\delta}{4(l+1)} \Lambda_{1}\left(V_{1}(x, t, k)\right)+\Lambda_{2}\left(V_{1}(x, t, k)\right) \phi_{2}\left(N_{1}(x, t, k)\right) & \forall x \in C
\end{array}
$$


hold for all $t \geq 0$ and $k \in \mathbb{Z}_{\geq 0}$. (This can be seen by bounding $\left|\Lambda_{1}^{\prime}\right|$ on the relevant intervals and recalling that $F, G \in \mathcal{U S B}$ and $V_{1} \in \mathcal{U P} \mathcal{P} \mathcal{D}$. In particular, to get (35), we write $\Delta_{k}\left(\Lambda_{1} \circ V_{5}\right)(x, t, k)=\Lambda_{1}^{\prime}\left(\eta V_{5}(F(x, k), t, k+1)\right.$ $\left.+(1-\eta) V_{5}(x, t, k)\right) \Delta_{k} V_{5}(x, t, k)$ for $\eta \in[0,1]$ depending on $x, t$, and $k$ and use the fact that $\Lambda_{1}^{\prime} \geq 0$ everywhere.) We next follow the remainder of the proof of Theorem 10 applied to $V_{6}$ for each $t \geq 0$ to get a function $V_{8}^{\text {dis }}(x, t, k)$ satisfying the conclusion of the proof when $x \in D$. We also apply the continuous time analog of that part of the proof to $V_{6}$ for each $k$ (with $\Delta_{k} V_{i}$ replaced by $\mathcal{D} V_{i}$ for all $i$ as before, similarly to the argument done in the Appendix below) to get $V_{8}^{\text {cts }}$ satisfying

$$
\mathcal{D} V_{8}^{\text {cts }}(x, t, k) \leq-\tilde{\alpha}(|x|), \quad \forall x \in C, t \geq 0, k \in \mathbb{Z}_{\geq 0}
$$

for a suitable $\tilde{\alpha} \in \mathcal{P} \mathcal{D}$. By enlarging $k_{4}, k_{5} \in \mathcal{K}_{\infty}$ and reducing $k_{3} \in \mathcal{P D}$ in the continuous and discrete versions of the proof, we can assume they are the same in both versions, so $V_{8}^{\text {cts }}$ and $V_{8}^{\text {dis }}$ have the same expression. Hence, we can satisfy the requirements of the theorem with their common value. Combined with the result of Lemma 6, this proves the theorem.

\section{Examples}

One class of systems covered by our discrete time results is as follows. Assume (1) is GAS and that a strict Lyapunov function $V$ for the system is available. This provides $\alpha_{1}, \alpha_{2} \in \mathcal{K}_{\infty}$ and $\alpha_{3} \in \mathcal{P} \mathcal{D}$ such that $\Delta_{k} V(x, k) \leq$ $-\alpha_{3}(|x|)$ and $\alpha_{1}(|x|) \leq V(x, k) \leq \alpha_{2}(|x|)$ everywhere. Assume now that the system is acted on by a PE term $p \in \mathcal{P}_{\mathrm{dis}}$ that freezes the dynamics for certain times. The new system becomes

$$
x_{k+1}=[1-p(k+1)] x_{k}+p(k+1) F\left(x_{k}, k\right) .
$$

Thus the new dynamic $F_{p}(x, k):=[1-p(k+1)] x+p(k+1) F(x, k)$ fixes the state when $p(k+1)=0$. By separately considering the cases $p(k+1)=0$ and $p(k+1)=1$, one checks that if $p(k) \in\{0,1\}$ for all $k$, then $V\left(F_{p}(x, k), k+1\right)-V(x, k) \leq-p(k+1) \alpha_{3}(|x|) \leq-p(k+1) \Theta(V(x, k))$ everywhere, where $\Theta(s)=\min \left\{\alpha_{3}(p): \alpha_{2}^{-1}(s) \leq p \leq \alpha_{1}^{-1}(s)\right\}$. Since $\Theta \in \mathcal{P} \mathcal{D}, V$ satisfies the PE decay condition from Theorem 7 for the new dynamic $F_{p}$. More generally, assume $p(k) \in[0,1]$ for all $k$. Assume also that $V(x, k)$ is a Lyapunov function for (1) that is independent of $k$ and convex in $x$. Choose $\alpha_{3} \in \mathcal{P D}$ such that $V(F(x, k))-V(x) \leq-\alpha_{3}(|x|)$ everywhere. Then

$$
\begin{aligned}
V\left(F_{p}(x, k)\right)-V(x) & \leq[1-p(k+1)] V(x)+p(k+1) V(F(x, k))-V(x) \\
& \leq-p(k+1) V(x)+p(k+1)\left[V(x)-\alpha_{3}(|x|)\right]=-p(k+1) \alpha_{3}(|x|)
\end{aligned}
$$

everywhere, so $F_{p}$ again satisfies our PE assumptions.

A general class of hybrid systems covered by our strictification results is as follows. Assume the continuous time system (2) admits $q \in \mathcal{P}_{\text {cts }}, \gamma \in \mathcal{K}_{\infty}, V \in C^{1}$, and $\alpha_{1}, \alpha_{2} \in \mathcal{K}_{\infty}$ satisfying $\mathcal{D} V(x, t) \leq-q(t) \gamma(V(x, t))$ and $\alpha_{1}(|x|) \leq V(x, t) \leq \alpha_{2}(|x|)$ for all $x \in \mathbb{R}^{n}$ and $t \geq 0$ (i.e., (2) admits a nonstrict Lyapunov function in the sense of [13]). ${ }^{1}$ Given subsets $C, D \subseteq \mathbb{R}^{n}$ and $p \in \mathcal{P}_{\text {dis }}$ taking all its values in $\{0,1\}$, we determine conditions on $F \in \mathcal{U S B}$ guaranteeing that we can construct a Lyapunov function for

$$
\mathcal{H}_{p}:= \begin{cases}\dot{x}=G(x, t), & x \in C \\ x_{k+1}=F_{p}\left(x_{k}, k\right), & x_{k} \in D\end{cases}
$$

where $F_{p}$ is as defined above. (The construction we are about to give also works if instead of assuming $p(k) \in\{0,1\}$ for all $k \in \mathbb{Z}_{\geq 0}$, we assume (i) $x \mapsto V(x, t)$ is convex for each $t \in[0, \infty)$ and (ii) $p(k) \in[0,1]$ for all $k \in \mathbb{Z}_{\geq 0}$. This situation arises if $\dot{x}=G(x, t):=A(t) x$ is GAS and $A(t)$ is continuous and bounded since then we can take $V(x, t):=x^{\top} P(t) x$ for a suitable bounded everywhere positive definite matrix $P(t)$ [10, Section 4.6].) To this end, first notice that by reducing $\gamma \in \mathcal{K}_{\infty}$ as necessary, we can assume $\gamma \in C^{1}$ and $\gamma(s) \leq \alpha_{1}\left(\alpha_{2}^{-1}(s)\right) / 2$ for all $s \geq 0$. Let $F$ satisfy $|F(x, k)| \leq \alpha_{2}^{-1}\left(\alpha_{1}(|x|) / 2\right)$ for all $x \in D$ and $k \in \mathbb{Z}_{\geq 0}$. (This reduces to a linear growth condition when

\footnotetext{
${ }^{1}$ A concrete example where this occurs and where it is easy to find $V$ is where $q \in \mathcal{P}_{\text {cts }}\left(\right.$ e.g. $\left.q(t)=\sin ^{2}(t)\right)$ and $\dot{x}=h(x, t)$ is GAS (e.g. $\dot{x}=-x)$ and we take the dynamic $G(x, t)=q(t) h(x, t)$ and a Lyapunov function $V(x, t)$ for $\dot{x}=h(x, t)$.
} 
$V(x, t)=x^{\top} P(t) x$ and $P$ has bounded positive eigenvalues.) By separately considering the cases $p(k+1)=0$ and $p(k+1)=1$, it follows that

$$
\begin{aligned}
V\left(F_{p}(x, k), t\right)-V(x, t) & \leq p(k+1) \alpha_{2}(|F(x, k)|)-p(k+1) \alpha_{1}(|x|) \\
& \leq-\frac{1}{2} p(k+1) \alpha_{1}(|x|) \leq-p(k+1) \gamma\left(\alpha_{2}(|x|)\right) \leq-p(k+1) \gamma(V(x, t))
\end{aligned}
$$

for all $x \in D, t \geq 0$, and $k \in \mathbb{Z}_{\geq 0}$. A slight variant of the proof of Theorem 11 therefore provides an explicit globally smooth strict Lyapunov function for $\mathcal{H}_{p}$ having the form

$$
V^{\sharp}(x, t, k):=2 V(x, t)+\frac{1}{\tau}\left[\int_{t-\tau}^{t} \int_{s}^{t} q(r) \mathrm{d} r \mathrm{~d} s\right] \gamma(V(x, t))+\left[\frac{1}{4(l+1)} \sum_{s=k-l}^{k} \sum_{j=s}^{k} p(j)\right] \gamma(V(x, t))
$$

for $l$ and $\tau$ as in the requirements $p \in \mathcal{P}_{\text {dis }}$ and $q \in \mathcal{P}_{\text {cts }}$ so $\mathcal{H}_{p}$ is GAS, as claimed.

\section{Conclusions}

We provided new methods for constructing closed form strict Lyapunov functions for hybrid systems that admit appropriate nonstrict Lyapunov functions. Our results cover cases where the given nonstrict Lyapunov functions satisfy a decay condition involving persistency of excitation parameters or hybrid versions of the conditions of Matrosov's Theorem. Due to the ubiquity of Lyapunov functions in engineering applications, we expect that our results will be useful in a wide range of settings in which explicit Lyapunov functions are needed such as Lyapunovbased controller design and robustness analysis. We conjecture that our results can be extended to hybrid control systems with outputs. This would extend $[25,26]$ and the input-to-output stability Lyapunov function constructions from [12] to hybrid systems and also provide more explicit constructions that would be suited for applications.

\section{Acknowledgements}

Michael Malisoff was supported by NSF Grant 0424011. He thanks Chaohong Cai, Rafal Goebel, Ricardo Sanfelice, and Andrew Teel for illuminating discussions at the 2005 American Control Conference in Portland, Oregon.

\section{Appendix}

Our proof of Theorem 14 was based on a continuous time version of Theorem 10. We next give a precise statement and proof of this continuous time result, which is applied in the proof of Theorem 14 to $(x, t) \mapsto V_{i}(x, t, k)$ for each $k$ and $i=1,2$. We assume the following version of the Matrosov conditions:

Assumption A.1. There exist $V_{1}: \mathbb{R}^{n} \times[0, \infty) \rightarrow[0, \infty)$ of class $\mathcal{U P} \mathcal{P} \mathcal{D}$ and $V_{2}: \mathbb{R}^{n} \times[0, \infty) \rightarrow \mathbb{R}$ of class $\mathcal{U S B}$ that are $C^{1}, \phi_{2} \in \mathcal{K}_{\infty}$, nonnegative functions $N_{1}, N_{2} \in \mathcal{U S B}$, a function $\chi: \mathbb{R}^{n} \times[0, \infty) \times[0, \infty) \rightarrow \mathbb{R}$, a positive increasing function $\phi_{1}, W \in \mathcal{P} \mathcal{D}$, and $q \in \mathcal{P}_{\text {cts }}$ such that

$$
\begin{aligned}
& \mathcal{D} V_{1}(x, t) \leq-N_{1}(x, t), \quad \mathcal{D} V_{2}(x, t) \leq-N_{2}(x, t)+\chi\left(x, N_{1}(x, t), t\right), \\
& \left|\chi\left(x, N_{1}(x, t), t\right)\right| \leq \phi_{1}(|x|) \phi_{2}\left(N_{1}(x, t)\right), \quad \text { and } \quad N_{1}(x, t)+N_{2}(x, t) \geq q(t) W(x)
\end{aligned}
$$

hold for all $x \in \mathbb{R}^{n}$ and $t \in[0, \infty)$.

Notice that $V_{2}$ can take both positive and negative values. We show:

Theorem A.2. If (2) satisfies Assumption A.1, then one can construct an explicit strict Lyapunov function for (2). In particular, (2) is GAS.

To prove this theorem, we indicate the changes needed in the proof of Theorem 10 . We define $V_{3}$ and $\lambda$ as in Section 5.1.2 which therefore satisfy

$$
\mathcal{D} V_{3}(x, t) \leq-q(t) W(x)+\phi_{1}(|x|) \phi_{2}\left(N_{1}(x, t)\right) \leq-q(t) \lambda\left(V_{1}(x, t)\right)+\phi_{1}(|x|) \phi_{2}\left(N_{1}(x, t)\right)
$$


everywhere. We also define $k_{1}, \Lambda_{1}$, and $V_{4}:=k_{1}\left(V_{1}\right) V_{3}$ as before and as before also determine positive increasing functions $\Gamma$ and $\Lambda_{2}$ such that

$$
\mathcal{D} V_{4}(x, t) \leq\left[-\mathcal{D} V_{1}(x, t)\right] \Gamma\left(V_{1}(x, t)\right)-q(t) \Lambda_{1}\left(V_{1}(x, t)\right)+\Lambda_{2}\left(V_{1}(x, t)\right) \phi_{2}\left(N_{1}(x, t)\right) .
$$

Choosing $k_{2} \in \mathcal{K}_{\infty}$ such that $k_{2}^{\prime} \geq \Gamma$ everywhere gives $\mathcal{D}\left(k_{2} \circ V_{1}\right)=k_{2}^{\prime}\left(V_{1}\right) \mathcal{D} V_{1} \leq \Gamma\left(V_{1}\right) \mathcal{D} V_{1}$, since $\mathcal{D} V_{1} \leq 0$ everywhere. Enlarging $k_{2} \in \mathcal{K}_{\infty}$ as necessary, it follows that $V_{5}:=V_{4}+k_{2}\left(V_{1}\right) \in \mathcal{U} \mathcal{P} \mathcal{P} \mathcal{D}$ satisfies

$$
\mathcal{D} V_{5}(x, t) \leq-q(t) \Lambda_{1}\left(V_{1}(x, t)\right)+\Lambda_{2}\left(V_{1}(x, t)\right) \phi_{2}\left(N_{1}(x, t)\right) .
$$

Applying the continuous time strictification method of [13] and enlarging $\Lambda_{2}$ and reducing $\Lambda_{1}$ as necessary without relabeling provides $\gamma \in \mathcal{K}_{\infty}$ and $\tau>0$ such that

$$
V_{6}(x, t):=V_{5}(x, t)+\left[\int_{t-\tau}^{t} \int_{s}^{t} q(r) \mathrm{d} r \mathrm{~d} s\right] \gamma\left(V_{5}(x, t)\right)
$$

satisfies $\mathcal{D} V_{6}(x, t) \leq-\Lambda_{1}\left(V_{1}(x, t)\right)+\Lambda_{2}\left(V_{1}(x, t)\right) \phi_{2}\left(N_{1}(x, t)\right)$. This uses the global boundedness of the double integral in (A.3) from Lemma 4. Arguing as in the proof of Theorem 10 gives $k_{3} \in \mathcal{P D} \cap C^{1}$ such that

$$
k_{3}(r) \leq \phi_{2}^{-1}\left(\frac{\Lambda_{1}(r)}{1+\Lambda_{2}(r)}\right) \frac{1}{1+\Lambda_{2}(r)}, \quad \text { hence } \phi_{2}\left(k_{3}\left(V_{1}\right) \Lambda_{2}\left(V_{1}\right)\right) \Lambda_{2}\left(V_{1}\right) \leq \Lambda_{1}\left(V_{1}\right)
$$

everywhere. Choose $k_{4} \in \mathcal{K}_{\infty} \cap C^{1}$ such that $k_{4}^{\prime}(s) \geq\left|k_{3}^{\prime}(s)\right|\left(\alpha_{6} \circ \alpha_{1}^{-1}\right)(s)$ everywhere, where $\alpha_{1}$ is as in the UPPD requirement on $V_{1}$, and $\alpha_{6}(|x|) \geq V_{6}(x, t)$ for all $x \in \mathbb{R}^{n}$ and $t \geq 0$. Then $k_{4}^{\prime}\left(V_{1}\right) \geq\left|k_{3}^{\prime}\left(V_{1}\right)\right| V_{6}$ everywhere, so $V_{7}:=k_{3}\left(V_{1}\right) V_{6}+k_{4}\left(V_{1}\right) \in \mathcal{U P} \mathcal{P} \mathcal{D}$ everywhere satisfies

$$
\begin{aligned}
\mathcal{D} V_{7} & \leq-k_{3}\left(V_{1}\right) \Lambda_{1}\left(V_{1}\right)+k_{3}\left(V_{1}\right) \Lambda_{2}\left(V_{1}\right) \phi_{2}\left(N_{1}\right)-V_{6}\left|k_{3}^{\prime}\left(V_{1}\right)\right| \mathcal{D} V_{1}+k_{4}^{\prime}\left(V_{1}\right) \mathcal{D} V_{1} \\
& \leq-k_{3}\left(V_{1}\right) \Lambda_{1}\left(V_{1}\right)+k_{3}\left(V_{1}\right) \Lambda_{2}\left(V_{1}\right) \phi_{2}\left(N_{1}\right) .
\end{aligned}
$$

The rest of the argument is similar to the corresponding part of the proof of Theorem 10 with $\Delta_{k}$ replaced by $\mathcal{D}$ and $\frac{\delta}{8(l+1)}$ replaced by 1 .

\section{References}

[1] F. Albertini, E.D. Sontag, Continuous control-Lyapunov functions for asymptotically controllable time-varying systems, International Journal of Control 72 (1999) 1630-1641.

[2] D. Angeli, Input-to-state stability of PD-controlled robotic systems, Automatica 35 (1999) 1285-1290.

[3] D. Angeli, E.D. Sontag, Forward completeness, unboundedness observability, and their Lyapunov characterizations, Systems and Control Letters 38 (1999) 209-217.

[4] D. Angeli, E.D. Sontag, Y. Wang, A characterization of integral input to state stability, IEEE Transactions on Automatic Control 45 (2000) 1082-1097.

[5] A. Bacciotti, L. Rosier, Liapunov Functions and Stability in Control Theory, Springer, London, 2001.

[6] C. Cai, A. Teel, R. Goebel, Converse Lyapunov theorems and robust asymptotic stability for hybrid systems, in: Proceedings of the 24th American Control Conference, Portand, OR, June 2005, pp. 12-17. http://www.ccec.ece.ucsb.edu/ cai/.

[7] P. Collins, A trajectory-space approach to hybrid systems, in: Proceedings of the International Symposium on the Mathematical Theory of Networks and Systems, Katholiek Univ. Leuven, Belgium, August 2004, Paper \#250, http://homepages.cwi.n1/ $/ \mathrm{collins} /$.

[8] J.-M. Coron, Global asymptotic stabilization for controllable systems without drift, Mathematics of Control, Signals \& Systems 5 (1992) 295-312.

[9] L. Faubourg, J.-B. Pomet, Control Lyapunov functions for homogeneous Jurdjevic-Quinn systems, ESAIM: Control, Optimisation and Calculus of Variations 5 (2000) 293-311.

[10] H. Khalil, Nonlinear Systems, Third ed., Prentice-Hall, Englewood Cliffs, NJ, 2002.

[11] M. Krichman, E.D. Sontag, Y. Wang, Input-output-to-state stability, SIAM Journal on Control and Optimization 39 (2001) 1874-1928.

[12] M. Malisoff, F. Mazenc, Further constructions of strict Lyapunov functions for time-varying systems, in: Proceedings of the American Control Conference, Portland, OR, June 2005, pp. 1889-1894.

[13] M. Malisoff, F. Mazenc, Further remarks on strict input-to-state stable Lyapunov functions for time-varying systems, Automatica 41 (2005) 1973-1978.

[14] J. Mancilla-Aguillar, R. Garcia, E.D. Sontag, Y. Wang, On the representation of switched systems with inputs by perturbed control systems, Nonlinear Analysis: Theory, Methods \& Applications 60 (2005) 1111-1150.

[15] J. Mancilla-Aguillar, R. Garcia, E.D. Sontag, Y. Wang, Uniform stability properties of switched systems with switchings governed by digraphs, Nonlinear Analysis: Theory, Methods \& Applications 63 (2005) 472-490. 
[16] F. Mazenc, Strict Lyapunov functions for time-varying systems, Automatica 39 (2003) 349-353.

[17] F. Mazenc, M. Malisoff, Further constructions of control-Lyapunov functions and stabilizing feedbacks for systems satisfying the Jurdjevic-Quinn conditions, IEEE Transactions on Automatic Control 51 (2006) 360-365.

[18] F. Mazenc, D. Nesić, Lyapunov functions for time varying systems satisfying generalized conditions of Matrosov theorem, in: Proceedings of the 44th IEEE Conference on Decision \& Control (CDC) \& European Control Conference ECC 05, Seville, Spain, December 2005, pp. 2432-2437.

[19] P. Morin, C. Samson, J.-B. Pomet, Design of homogeneous time-varying stabilizing control laws for driftless systems via oscillatory approximation of Lie brackets in closed loop, SIAM Journal on Control and Optimization 38 (1999) 22-49.

[20] D. Nesić, A. Loria, On uniform asymptotic stability of time-varying parameterized discrete-time cascades, IEEE Transactions on Automatic Control 49 (2004) 875-887.

[21] D. Nesić, A. Teel, E.D. Sontag, Formulas relating $\mathcal{K} \mathcal{L}$ stability estimates of discrete-time and sampled-time nonlinear systems, Systems and Control Letters 38 (1999) 49-60.

[22] C. Samson, Velocity and torque feedback control of a nonholonomic cart, in: Advanced Robot Control (Grenoble, 1990), in: Lecture Notes in Control and Information Sciences, vol. 162, Springer, Berlin, 1991, pp. 125-151.

[23] E.D. Sontag, Smooth stabilization implies coprime factorization, IEEE Transactions on Automatic Control 34 (1989) $435-443$.

[24] E.D. Sontag, Feedback stabilization of nonlinear systems, in: M.A. Kaashoek (Ed.), Robust Control of Linear Systems and Nonlinear Control, Birkhäuser, Basel, 1990, pp. 61-81.

[25] E.D. Sontag, Y. Wang, Notions of input to output stability, Systems and Control Letters 38 (1999) 235-248.

[26] E.D. Sontag, Y. Wang, Lyapunov characterizations of input to output stability, SIAM Journal on Control and Optimization 39 (2001) $226-249$.

[27] A. Van der Schaft, H. Schumacher, An Introduction to Hybrid Systems, in: Lecture Notes in Control and Information Sciences, vol. 251, Springer-Verlag, London, 2000. 